\title{
Thermopower and Hardness Characterization of Structural Relaxation and Crystallization in FINEMET Type Amorphous Precursor Alloy
}

\author{
K. BÁN ${ }^{a}, \mathrm{~A} . \mathrm{SzABÓ}^{a, b, *}, \mathrm{R} . \mathrm{IPACH}^{a}$ AND B. SzABÓ ${ }^{a}$ \\ ${ }^{a}$ Department of Automobiles and Vehicle Manufacturing, Budapest University of Technology and Economics, \\ Stoczek Str. 6, 1111 Budapest, Hungary \\ ${ }^{b}$ Institute of Engineering, University of Dunaujvaros, Tancsics M. Str. 1/a, 2401 Dunaujvaros, Hungary \\ In this paper, the thermopower $(\mathrm{U})$ and hardness $(\mathrm{HV})$ of FINEMET type glasses are studied during structu- \\ ral relaxation and beyond the crystallization onset. In this multicomponent alloy $\left(\mathrm{Fe}_{73.5-x} \mathrm{Si}_{13.5} \mathrm{~B}_{9} \mathrm{Nb}_{x} \mathrm{Cu}_{1}\right)$ both \\ property changes are more complex than that in binary $\mathrm{Fe}-\mathrm{B}$ glasses. The phenomenon of relaxation and the \\ crystallization onset can be successfully distinguished in both property changes. Simultaneously with the hardness \\ increase, a slope increase in the thermopower versus temperature dependences was observed if crystallization star- \\ ted. The character of both property changes does alter drastically at the beginning of amorphous-nanocrystalline \\ transformation. Similar trends were measured on samples undergoing traditional isothermal and pulse heat treat- \\ ments.
}

DOI: 10.12693/APhysPolA.131.702

PACS/topics: 61.43.Dq, 72.15.Jf

\section{Introduction}

Recently the measurement of transport properties such as thermopower $(U(T))$ or electrical resistance $(\rho(T))$ - have been successfully applied for the study of phase transformations as well as monitoring the inner stress state, in various metallic glassy systems [1-3]. The method has also been applied for monitoring the degree of structural relaxation as well as for the study of amorphous-crystalline transition [4]. However, for the detection of inner stress state and structural relaxation the measurement of thermopower is not sufficient and for this reason additional methods like the investigation of the Curie temperature shift and the measurement of hardness change are also often used $[5,6]$.

The study of amorphous-crystalline transformation using thermopower measurements is performed predominantly in compositionally simple systems. Here the shift of the $U(T)$ slope attributed to the individual components can be recognized easily.

The compositionally complex FINEMET alloy was chosen for this study in order to detect not only the early stage of relaxation but also the possible overlapping of the relaxation effect and the early stage of the primary crystallization (precipitation of $\alpha-\mathrm{Fe}(\mathrm{Si})$ ) to which extraordinary magnetic softness of the FINEMET type alloys is attributed.

The thermopower and microhardness measurements were carried out simultaneously in order to detect mechanical hardening and magnetic softening in initial state of transformation in this type of alloy.

*corresponding author; e-mail: szabo.attila@uniduna.hu

\section{Experiments}

Isothermal and pulse annealing were performed on FINEMET-type $\left(\mathrm{Fe}_{73.5-x} \mathrm{Si}_{13.5} \mathrm{~B}_{9} \mathrm{Nb}_{x} \mathrm{Cu}_{1}\right)$ amorphous ribbons. The parameters of heat treatments are listed in Table I. The details of pulse heat treatments are described in [7]. Impulse heating was performed by alternating the current pulses of approximately $10 \mathrm{~A}, 50 \mathrm{~Hz}$. Pulse duration was $0.3-0.5 \mathrm{~s}$. Samples were processed under this heat without applying forced air cooling. So, the estimated cooling rate is about $100 \mathrm{~K} / \mathrm{s}$. Thermal heating rate was about $1000 \mathrm{~K} / \mathrm{s}$.

TABLE I

Heat treatment parameters.

\begin{tabular}{c|c|c|c}
\hline \hline \multicolumn{4}{c}{ Isothermal heat treatment (temp./time) } \\
\hline $240{ }^{\circ} \mathrm{C} / 1 \mathrm{~h}$ & $240{ }^{\circ} \mathrm{C} / 5 \mathrm{~h}$ & $290{ }^{\circ} \mathrm{C} / 1 \mathrm{~h}$ & $340{ }^{\circ} \mathrm{C} / 1 \mathrm{~h}$ \\
$390{ }^{\circ} \mathrm{C} / 1 \mathrm{~h}$ & $450{ }^{\circ} \mathrm{C} / 1 \mathrm{~h}$ & $500{ }^{\circ} \mathrm{C} / 1 \mathrm{~h}$ & $540{ }^{\circ} \mathrm{C} / 1 \mathrm{~h}$ \\
\hline \multicolumn{4}{c}{ Pulse heat treatment at $240{ }^{\circ} \mathrm{C}$} \\
(pulse number $\times$ pulse length $[\mathrm{s}]$ ) \\
\hline $1 \times 0.1$ & $3 \times 0.1$ & $5 \times 0.1$ & $10 \times 0.1$
\end{tabular}

\section{Results and discussion}

Thermal activation enables only short-range atomic displacements during structural relaxation, involving short-range reorientation of chemical bonds between the neighboring atoms. Consequently, atomic diffusion over several atomic distances - which results in a critical nuclei formation of any crystalline phases - does not occur.

Nevertheless, the structural stabilization which is detectable as a small volume contraction (bulk densification) hints to the existence of thermodynamic stabilization related to the as-quenched state. This phenomenon is also reflected in the change of electronic structure. 
In Fig. 1, the $U(T)$ curves for FINEMET alloy are plotted after various isothermal annealing. If the heat treatment temperature is low (lower than the glass transition temperature, $T_{g}$ ), structural relaxation proceeds only, hence, thermally induced stabilization of the frozen glassy state takes place. The small shift of the $U(T)$ plot is regarded as the manifestation of the glassy state stabilization (the slope of the curve slightly changes, but this effect is within the experimental error). This effect predicts negligible change in the free energy of the glassy state (heat treatments at 240 and $340{ }^{\circ} \mathrm{C}$ in Fig. 1). Also small change in electrical conductivity is expected according to the Mott approximation [8], because mobility of conduction electrons at the Fermi surface slightly decreases due to the relaxation. In contrast, at the beginning of crystallization (when crystallization starts) the slope of the $U(T)$ curve shifts significantly to the opposite (positive) direction, i.e., the slope simultaneously decreases.

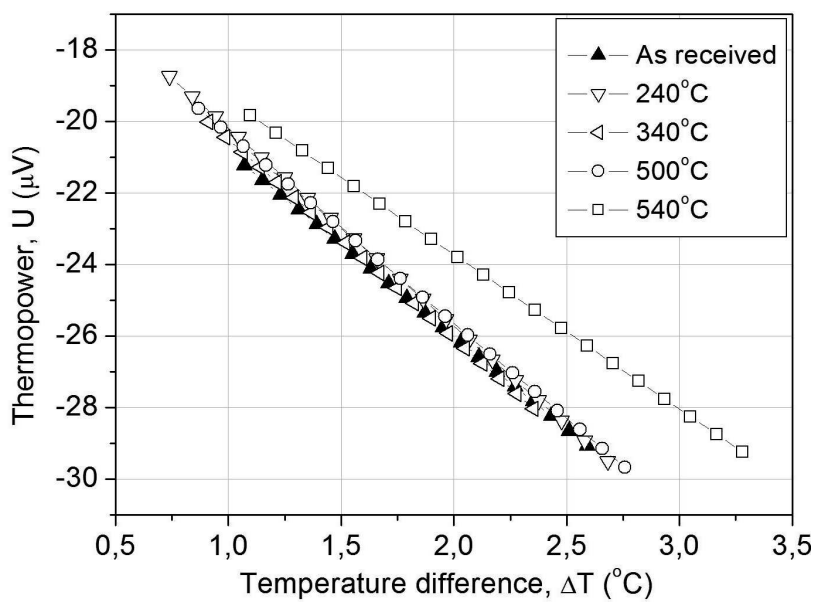

Fig. 1. Thermopower of FINEMET alloy after various isothermal $(1 \mathrm{~h})$ heat treatments.

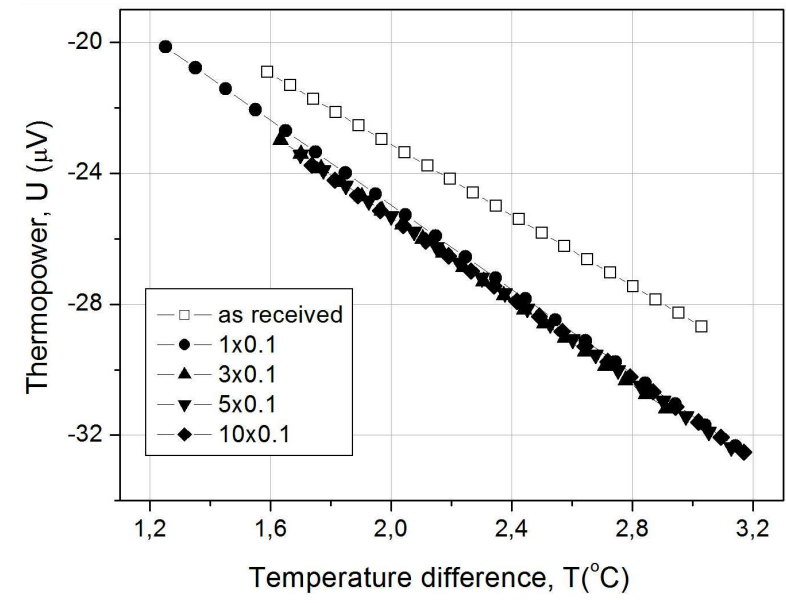

Fig. 2. The thermopower shift after increase of the number of $0.10 \mathrm{~s}$ length pulses (peak temperature $\left.240^{\circ} \mathrm{C}\right)$.

The crystallization mechanisms of FINEMET glasses are similar to that in the hypoeutectic Fe-B, which in- dicate a two-step mechanism. The first step is the $\alpha$-Fe precipitation. The sign of appropriate shift (heat treatment at $540^{\circ} \mathrm{C}$ ) is in qualitative agreement with the experimentally detected $\alpha-\mathrm{Fe}(\mathrm{Si})$ nanograin precipitation from glassy matrix [9].

The results depicted in Fig. 1 are qualitatively supported by the series of pulse heat treatments summarized in Fig. 2, where the shift of $U(T)$ curves are illustrated versus the number of $0.10 \mathrm{~s}$ length pulses (small, increasing slope). According to [7] the peak temperature during the applied pulses is $345^{\circ} \mathrm{C}$. Solely structural relaxation occurs at this temperature.

\subsection{The hardness evolution during isothermal and pulse annealings}

The hardness increase of transition metal-metalloid (TM-M) glasses during relaxation annealing is widely known [3]. According to the literature, this increase occurs slightly below $400^{\circ} \mathrm{C}$ as Fig. 3 shows. The figure indicates that slope of $\mathrm{HV}$ increase depends primarily on the temperature of heat treatment and only slightly depends on the heat treatment duration, showing the significance of thermal activation short range rearrangements involved. The abrupt increase indicates the beginning of crystallization, which is in agreement with structural investigation [9].

Similar tendency can be recognized during the pulse annealing as well (see Fig. 4).

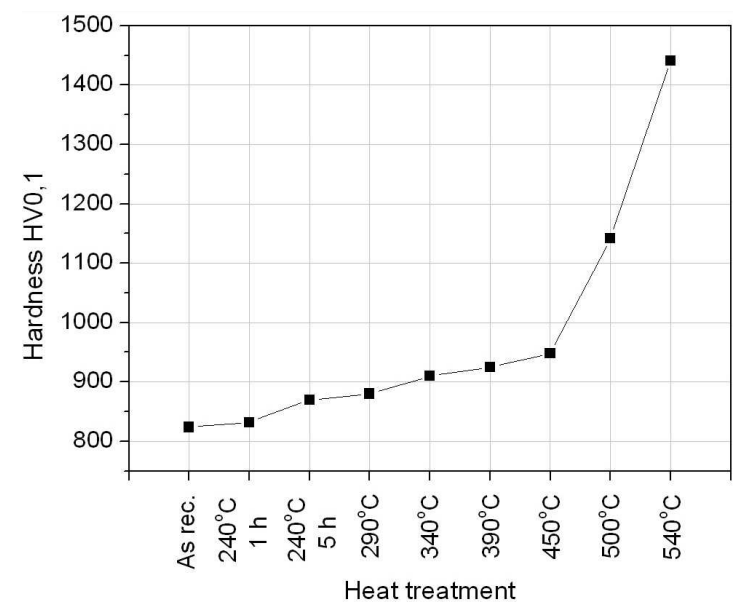

Fig. 3. The hardness evolution of FINEMET glass versus the increase of temperature of isotherm $(1 \mathrm{~h})$ heat treatments (the role of heat treatment duration has secondary importance).

The increase in hardness is demonstrated in Fig. 4, versus the pulse length after different numbers of applied pulses. According to [7] the increase of pulse length represents also increase of peak temperature. Below the critical pulse length $\left(t_{s}<0.08 \mathrm{~s}\right)$ the resulting hardness increase is within the experimental error.

If the pulse length is $0.08 \mathrm{~s}$ (peak temperature exceeds $240{ }^{\circ} \mathrm{C}$ ) or longer, then the hardness suddenly increases (note that higher hardness values are measured after the pulse heat treatments). 


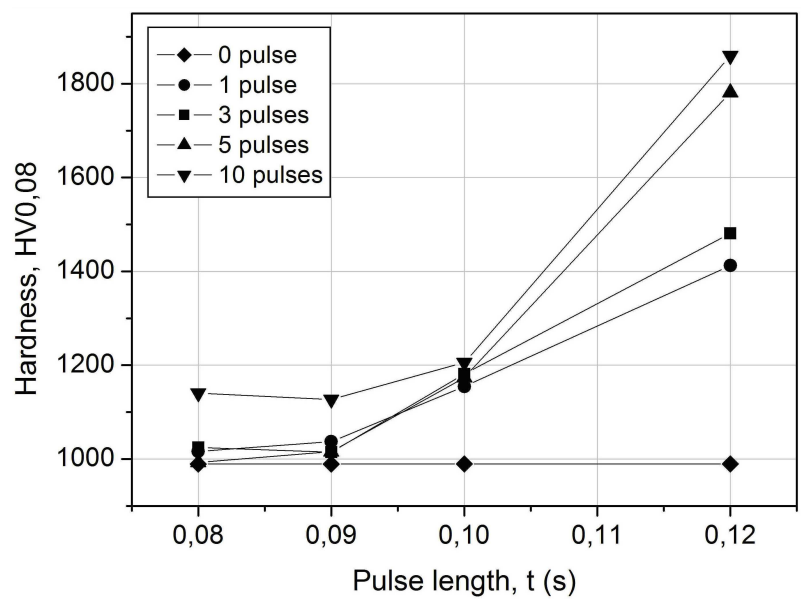

Fig. 4. Hardness versus increasing pulse length (the curves refer to various pulse number at $240{ }^{\circ} \mathrm{C}$ peak temperature heat treatments).

The significant difference between the hardness results plotted in Fig. 3 and Fig. 4 may arise partly from different heat treatment method applied in the two series of measurements [10].

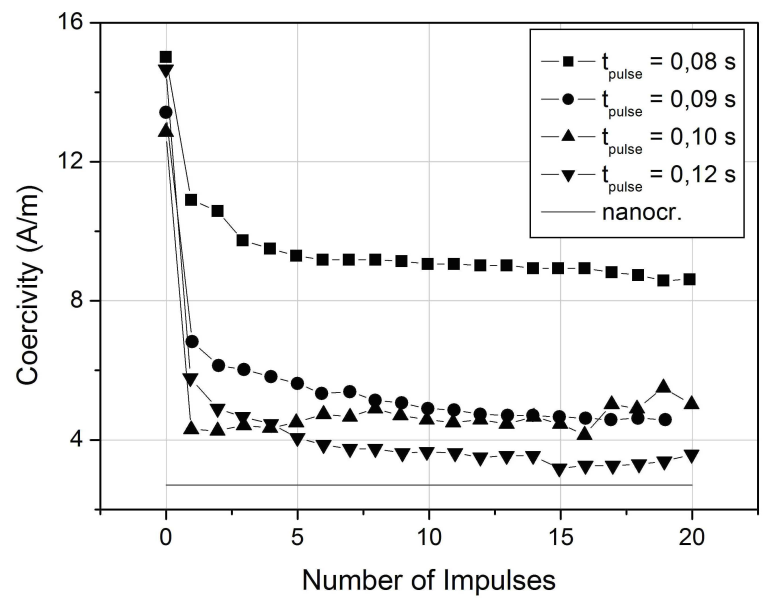

Fig. 5. Coercivity of FINEMET samples versus the pulse number, applying increasing pulse length. Peak temperature $240^{\circ} \mathrm{C}$ (figure published with kind permission of Dr. Novák, Technical University of Košice).

An important observation is that the thermal activation for the thermopower and hardness change is significantly different. As Fig. 2 shows, the $U(T)$ sensitively changes even due to a single pulse. The decrease in the coercive force is similar, as it is clearly seen in Fig. 5, the $H_{c}$ decrease is nearly completed even due to a single pulse (peak temperature at $\left.240^{\circ} \mathrm{C}\right)$. This finding indicates the differences in the relaxation mechanism of these properties.
For the $U(T)$ and $H_{c}$ relaxation the dominant contribution arises from the change of electronic structure, while the bond reorientation and short range atomic displacements cannot be excluded in the hardness change.

\section{Conclusion}

1. The thermopower and hardness change in FINEMET type alloys are compared during isothermal and pulse heat treatments, leading to various degree of structural relaxation and partial crystallization.

2. Both the thermopower and hardness change are small in the period of structural relaxation and an abrupt change can be detected at the crystallization onset.

3 . In contrast to the hardness change, the thermopower is more sensitive to the small temperature increase. This property is also supported by the coercivity change in the same alloy.

4. Based on the experimental results, it is assumed that low thermal activation is required for the thermopower change, as dominantly electronic rearrangements is involved in this process. Similarly, the rapid decrease in coercivity during the short time-scale pulse heat treatments also hints the non-diffusive nature of coercivity change during the relaxation processes. In contrast, a short atomic scale rearrangement is assumed to be involved in the hardness increase.

\section{References}

[1] Z. Pál, A. Lovas Acta Phys. Pol. A 113, 139 (2008).

[2] Z. Pál, J. Takács, Period. Polytech. Transp. Eng. 35, 65 (2007).

[3] Cs. Gulyás, A. Lovas, Period. Polytech. Transp. Eng. 32, 91 (2004).

[4] A. Szabo, K. Varga, A. Lovas, in: Int. Colloq. of Advanced Manufacturing and Repair Technologies in Vehicle Industry, Eds.: J. Takács, T. Markovits, B. Vehovszky, Budapest University of Technology and Economics, Budapest 2013, p. 29.

[5] A. Lovas, Acta Phys. Pol. A 118, 770 (2008).

[6] J.O. Ström-Olsen, R. Brüning, Z. Altounian, D.H. Ryan, J. Less Common Met. 145, 327 (1988).

[7] J. Kovác, L. Novák, L. Hubac, J. Electr. Eng. 66, 142 (2015).

[8] S.N. Mott, Conduction in Non-Crystalline Materials, Clarendon Press, Oxford 1993.

[9] K. Hono, K. Hiraga, Q. Wang, A. Inoue, T. Sakurai, Acta Metall. Mater. 40, 2137 (1992).

[10] Z. Pál, PhD Thesis, Budapest University of Technolgy and Economics, Budapest 2010. 